\title{
Microwave-Assisted Synthesis of Spirofused Heterocycles Using Decatungstodivanadogermanic Heteropoly Acid as a Novel and Reusable Heterogeneous Catalyst under Solvent-Free Conditions
}

\author{
Srinivasa Rao Jetti, Divya Verma, and Shubha Jain \\ Laboratory of Heterocycles, School of Studies in Chemistry and Biochemistry, Vikram University, Ujjain 456010, \\ Madhya Pradesh, India
}

Correspondence should be addressed to Srinivasa Rao Jetti; srinujetti479@gmail.com

Received 8 October 2012; Accepted 7 January 2013

Academic Editor: Mohammed M. Bettahar

Copyright (C) 2013 Srinivasa Rao Jetti et al. This is an open access article distributed under the Creative Commons Attribution License, which permits unrestricted use, distribution, and reproduction in any medium, provided the original work is properly cited.

Decatungstodivanadogermanic acid $\left(\mathrm{HH}_{6} \mathrm{GeW}_{10} \mathrm{~V}_{2} \mathrm{O}_{40} \cdot 22 \mathrm{H}_{2} \mathrm{O}\right)$ was synthesized and used as a novel, green heterogeneous catalyst for the synthesis of spirofused heterocycles from one-pot three-component cyclocondensation reaction of a cyclic ketone, aldehyde, and urea in high yields under solvent-free condition in microwave irradiation at $80^{\circ} \mathrm{C}$. This catalyst is efficient not only for cyclic ketones, but also for cyclic $\beta$-diketones, $\beta$-diester, and $\beta$-diamide derivatives such as cyclohexanone, dimedone, and Meldrum's acid, or barbituric acid derivatives.

\section{Introduction}

Dihydropyrimidinones and their derivatives have attracted great attention recently in synthetic organic chemistry due to their pharmacological and therapeutic properties such as antibacterial and antihypertensive activity as well as behaving as calcium channel blockers, $\alpha$-la-antagonists [1], and neuropeptide Y (NPY) antagonists [2]. The biological activity of some alkaloids isolated recently has been attributed to a dihydropyrimidinone moiety [3]. The first procedure to these compounds reported by Biginelli [4] more than a century ago makes use of the three-component, one-pot condensation of a $\beta$-ketoester, an aldehyde, and a urea under strongly acidic conditions [4]. However this method suffers from low yields in the case of substituted aromatic and aliphatic aldehydes [5]. Owing to the versatile biological activity of dihydropyrimidinones, development of an alternative synthetic methodology is of paramount importance.

Recently, many reviews $[8,9]$ and papers for preparing these compounds have been reported including classical conditions, with microwave and ultrasound irradiation and by using some other different catalysts such as phosphorus pentoxide-methanesulfonic acid [10], potassium terbutoxide
( $t$-BuOK) [11], ammonium dihydrogen phosphate [12], silicagel [13], mesoporous molecular sieve MCM-41 [14], cyanuric chloride [15], nano- $\mathrm{BF}_{3} \cdot \mathrm{SiO}_{2}$ [16], silica gel-supported polyphosphoric Acid [17], zirconium(IV) chloride [18], indium(III) bromide [19], ytterbium(III)-resin [20], 1- $n$ butyl-3-methylimidazolium tetrafluoroborate $\left(\mathrm{BMImBF}_{4}\right)$ or hexafluorophosphorate $\left(\mathrm{BMImPF}_{6}\right)[21]$, ceric ammonium nitrate (CAN) [22], $\mathrm{Mn}(\mathrm{OAc})_{3} \cdot 2 \mathrm{H}_{2} \mathrm{O}$ [23], lanthanide triflate [24], indium(III) chloride [25], lanthanum chloride [26], $\mathrm{H}_{2} \mathrm{SO}_{4}$ [27], montmorillonite KSF [28], polyphosphate ester (PPE) [29], BF3-OEt ${ }_{2} / \mathrm{CuCl} / \mathrm{HOAc}$ [30], and conc. $\mathrm{HCl}$ $[31,32]$.

However, in spite of their potential utility, many of these methods involve expensive reagents, strongly acidic conditions, long reaction times, high temperatures, and stoichiometric amounts of catalysts and give unsatisfactory yields. Therefore, the discovery of a new catalyst for the preparation of pyrimidinones under neutral and mild conditions is of prime importance. Heterogeneous acid catalysis by heteropoly acids (HPAs) has attracted much interest because of its potential of great economic rewards and green benefits [3335]. Unlike metal oxides and zeolites, HPAs possess very strong Bronsted acidity, and their acid sites are more uniform 
and easier to control than those in other solid acid catalysts. These catalysts make them suitable solid heterogeneous catalysts for organic transformations.

Microwave reaction under solvent-free conditions and/or in the presence of a catalyst, resulting in shorter reaction time and higher product yields than those obtained by using conventional heating, offer low cost together with simplicity in processing and handling [36]. In connection with our previous works on synthesis of pyrimidinones derivatives [3739] and Meldrum's acid and barbituric acid derivatives [40], we wish to report the results obtained from a study of the reaction of aldehydes, urea, cyclohexanone, and Meldrum's acid or barbituric acid derivatives as a $\mathrm{CH}$-acid, instead of open-chain cyclic $\beta$-dicarbonyl compounds, in microwave irradiation under solvent-free conditions. The procedure not only gives products in good yields but also avoids problems connected with solvent use (cost, handling, safety, and pollution), and the reaction times.

\section{Experimental}

2.1. Materials and Methods. All reactions were carried out in an LG domestic unmodified microwave oven model MS-1947C/01. Melting points were measured on an Electrothermal 9100 apparatus and are uncorrected. Mass spectra were recorded on a FINNIGAN-MAT 8430 mass spectrometer operating at an ionization potential of $70 \mathrm{eV}$. IR spectra were recorded on a Shimadzu IR-470 spectrometer. ${ }^{1} \mathrm{H}$ and ${ }^{13} \mathrm{C}$ NMR spectra were recorded on a BRUKER DRX-500 AVANCE spectrometer at 500.13 and $125.77 \mathrm{MHz}$, respectively. NMR spectra were obtained on solutions in DMSO- $d_{6}$. The chemicals used in this work were purchased from Fluka (Buchs, Switzerland) Chemical Company. Decatungstodivanadogermanic acid $\left(\mathrm{H}_{6} \mathrm{GeW}_{10} \mathrm{~V}_{2} \mathrm{O}_{40} \cdot 22 \mathrm{H}_{2} \mathrm{O}\right)$ was prepared according to a reported procedure [41].

2.2. Synthesis of Catalyst. $0.8 \mathrm{~g}$ of $\mathrm{GeO}_{2}$ was dissolved in a hot solution of $10 \% \mathrm{NaOH}$, and a solution of $22.8 \mathrm{~g}$ of $\mathrm{Na}_{2} \mathrm{WO}_{4}$. $2 \mathrm{H}_{2} \mathrm{O}$ in $100 \mathrm{~mL}$ of hot water was added to get mixture A. The $\mathrm{pH}$ of $\mathrm{A}$ was adjusted to 6 with $\mathrm{HCl}(1: 1)$ and heated for $1 \mathrm{~h}$. Then a solution of $7.5 \mathrm{~g}$ of $\mathrm{Na}_{2} \mathrm{CO}_{3}$ dissolved in $25 \mathrm{~mL}$ of hot water was added. The mixture was concentrated to $100 \mathrm{~mL}$ by heating. $2.4 \mathrm{~g}$ of $\mathrm{NaVO}_{3} \cdot 2 \mathrm{H}_{2} \mathrm{O}$ and $2.5 \mathrm{~g}$ of $\mathrm{Na}_{2} \mathrm{WO}_{4} \cdot 2 \mathrm{H}_{2} \mathrm{O}$ were dissolved in $30 \mathrm{~mL}$ of hot water, respectively, and the two solutions were mixed to get mixture $\mathrm{B}$. The $\mathrm{pH}$ of mixture $\mathrm{B}$ was adjusted to 2.5 with $\mathrm{H}_{2} \mathrm{SO}_{4}(1: 1)$. Then $\mathrm{A}$ was added dropwise, and the $\mathrm{pH}$ was kept at 2.5 while dropping. After stirring for $3 \mathrm{~h}$ at $60^{\circ} \mathrm{C}$, the solution was cooled to room temperature. The cooled solution was extracted with ether in sulfuric acid medium, and the extractant was dissolved with a small amount of water. After the ether was evaporated, the remaining mixture was placed in the desiccators until orange crystals were separated out. The final yield was about $70 \%$. Anal. Calcd. for $\mathrm{H}_{6} \mathrm{GeW}_{10} \mathrm{~V}_{2} \mathrm{O}_{40} \cdot 22 \mathrm{H}_{2} \mathrm{O}$ : Ge, 2.38; W, 60.18; $\mathrm{V}, 3.33 ; \mathrm{H}_{2} \mathrm{O}, 12.96$. Found: $\mathrm{Ge}, 2.38 ; \mathrm{W}, 60.06 ; \mathrm{V}, 3.29 ; \mathrm{H}_{2} \mathrm{O}$, $12.97 \%$ (TG analysis). FT-IR $\left(\mathrm{KBr}, \mathrm{cm}^{-1}\right): 3450 v(\mathrm{O}-\mathrm{H})$; $1620 \delta(\mathrm{O}-\mathrm{H}) ; 964 v_{\mathrm{as}}\left(\mathrm{M}-\mathrm{O}_{\mathrm{d}}\right) ; 885 v_{\mathrm{as}}\left(\mathrm{M}-\mathrm{O}_{\mathrm{b}}-\mathrm{M}\right) ; 818 v_{\mathrm{as}}$ $\left(\mathrm{Ge}-\mathrm{O}_{\mathrm{a}}\right) ; 780 v_{\mathrm{as}}\left(\mathrm{M}-\mathrm{O}_{\mathrm{c}}-\mathrm{M}\right) ; 464 \delta(\mathrm{O}-\mathrm{Ge}-\mathrm{O}),(\mathrm{M}=\mathrm{W}$ and
$\mathrm{V} ; \mathrm{O}_{\mathrm{a}}$, inner oxygen; $\mathrm{O}_{\mathrm{b}}$, corner-shared oxygen; $\mathrm{O}_{\mathrm{c}}$, edgeshared oxygen; $\mathrm{O}_{\mathrm{d}}$, terminal oxygen) [41]. UV-Vis spectrum $\left(\mathrm{CH}_{3} \mathrm{CN} \lambda_{\max } \mathrm{nm}\right) ;\left(\mathrm{O}_{\mathrm{d}} \rightarrow \mathrm{M}, \mathrm{CT}\right) ; 262\left(\mathrm{O}_{\mathrm{b} / \mathrm{c}} \rightarrow \mathrm{M}, \mathrm{CT}\right)$.

The number of hydrogen in the HPA and the states of ionization can be determined by potentiometric titration. The potentiometric titration curve (Figure 1) shows that the six protons of $\mathrm{H}_{6} \mathrm{GeW}_{10} \mathrm{~V}_{2} \mathrm{O}_{40} \cdot 22 \mathrm{H}_{2} \mathrm{O}$ are equivalent and they are ionized in one step.

$\mathrm{X}$-ray powder diffraction is widely used to study the structural features of HPA and explain their properties [42]. The data of X-ray powder diffraction are listed in Table 1.

The result of $\mathrm{X}$-ray powder diffraction of $\mathrm{H}_{6} \mathrm{GeW}_{10}$ $\mathrm{V}_{2} \mathrm{O}_{40} \cdot 22 \mathrm{H}_{2} \mathrm{O}$ displays that the diffraction peaks are primarily distributed in four ranges of $2 \theta$ which are $7-10^{\circ}, 16-22^{\circ}$, $25-30^{\circ}$, and $33-38^{\circ}$. The positions and intensities of the main peaks are similar to those expected for the Keggin structure. Combined with IR and UV spectra, it is sure that $\mathrm{H}_{6} \mathrm{Ge}$ $\mathrm{W}_{10} \mathrm{~V}_{2} \mathrm{O}_{40} \cdot 22 \mathrm{H}_{2} \mathrm{O}$ possesses Keggin structure.

HPA consists of protons, HPA anions, and hydration water. Figure 2 is the thermogram of $\mathrm{H}_{6} \mathrm{GeW}_{10} \mathrm{~V}_{2} \mathrm{O}_{40}$. $22 \mathrm{H}_{2} \mathrm{O}$. The TG curve shows that the total percent of weight loss is $12.96 \%$, which indicates that each HPA molecule has 22 molecules of water, and there are three steps of weight loss. The first is the loss of 16 molecules of hydration water, the second is the loss of 6 molecules of protonized water and the third is the loss of 3 molecules of structural water. Thus, the accurate molecular formula of the product is $\left(\mathrm{H}_{5} \mathrm{O}_{2}\right)$ $3 \mathrm{H}_{3} \mathrm{GeW}_{10} \mathrm{~V}_{2} \mathrm{O}_{40} \cdot 16 \mathrm{H}_{2} \mathrm{O}$.

In general, we took the temperature of the exothermic peak of DTA curves as a sign of their thermostability [43]. In the DTA curve, there was an exothermic peak at $481.6^{\circ} \mathrm{C}$.

\subsection{General Procedure for the Reaction of Benzaldehyde Mel-} drum's Acid and Urea. An intimate mixture of benzaldehyde $(0.30 \mathrm{~g}, 2 \mathrm{mmol})$, Meldrum's acid $(0.144 \mathrm{~g}, 1 \mathrm{mmol})$, urea $(0.06 \mathrm{~g}, 1 \mathrm{mmol})$, and decatungstodivanadogermanic acid $(0.03 \mathrm{~g} 3 \mathrm{mmol})$ was subjected to microwave irradiation for appropriate time in $600 \mathrm{~W}$ microwave oven for 6-7 min (successive irradiation of 30-40 sec with cooling intervals of time as the temperature being $80^{\circ} \mathrm{C}$ ) as indicated by TLC. After cooling, $\mathrm{H}_{6} \mathrm{GeW}_{10} \mathrm{~V}_{2} \mathrm{O}_{40} \cdot 22 \mathrm{H}_{2} \mathrm{O}$ was separated by simple filtration due to its heterogeneous nature, and the reaction mixture was poured onto crushed ice $(40 \mathrm{~g})$ and stirred for 5-10 min. The precipitate was filtered under suction, washed with cold water $(40 \mathrm{~mL})$ and ethyl acetate $(5 \mathrm{~mL})$ to afford the pure product 1a.

2.4. General Procedure for the Reaction of Cyclohexanone, Aldehydes, and Urea. The mixture of cyclohexanone $(1.0 \mathrm{mmol})$, aldehyde $(2.0 \mathrm{mmol})$, urea $(3.0 \mathrm{mmol})$, and Decatungstodivanadogermanic acid $(3 \mathrm{mmol})$ was subjected to microwave irradiation for appropriate time in $600 \mathrm{~W}$ microwave oven for 6-7 min (successive irradiation of 30-40 sec with cooling intervals of time as the temperature being $80^{\circ} \mathrm{C}$ ) as indicated by TLC. After cooling, $\mathrm{H}_{6} \mathrm{GeW}_{10} \mathrm{~V}_{2} \mathrm{O}_{40} \cdot 22 \mathrm{H}_{2} \mathrm{O}$ was separated by simple filtration due to its heterogeneous nature and the reaction mixture was poured onto crushed ice ( $40 \mathrm{~g}$ ) and stirred for 5-10 $\mathrm{min}$. The precipitate was filtered 
TABle 1: Data of X-ray powder diffraction of $\mathrm{H}_{6} \mathrm{GeW}_{10} \mathrm{~V}_{2} \mathrm{O}_{40} \cdot 22 \mathrm{H}_{2} \mathrm{O}$.

\begin{tabular}{lccccccc}
\hline $2 \theta /^{\circ}$ & 9.27 & 10.34 & 16.76 & 18.75 & 19.10 & 20.76 & 25.52 \\
$\mathrm{~d} / \mathrm{nm}$ & 0.954 & 0.855 & 0.529 & 0.473 & 0.465 & 0.428 & 0.349 \\
$\mathrm{I}$ & 95.8 & 100.0 & 14.6 & 25.0 & 47.9 & 41.7 & 45.8 \\
$2 \theta /^{\circ}$ & 27.09 & 28.00 & 29.57 & 34.70 & 35.40 & 36.72 & 37.79 \\
$\mathrm{~d} / \mathrm{nm}$ & 0.329 & 0.319 & 0.302 & 0.529 & 0.254 & 0.245 & 0.238 \\
$\mathrm{I}$ & 70.8 & 60.4 & 27.1 & 33.3 & 22.9 & 35.4 & 27.1 \\
\hline
\end{tabular}

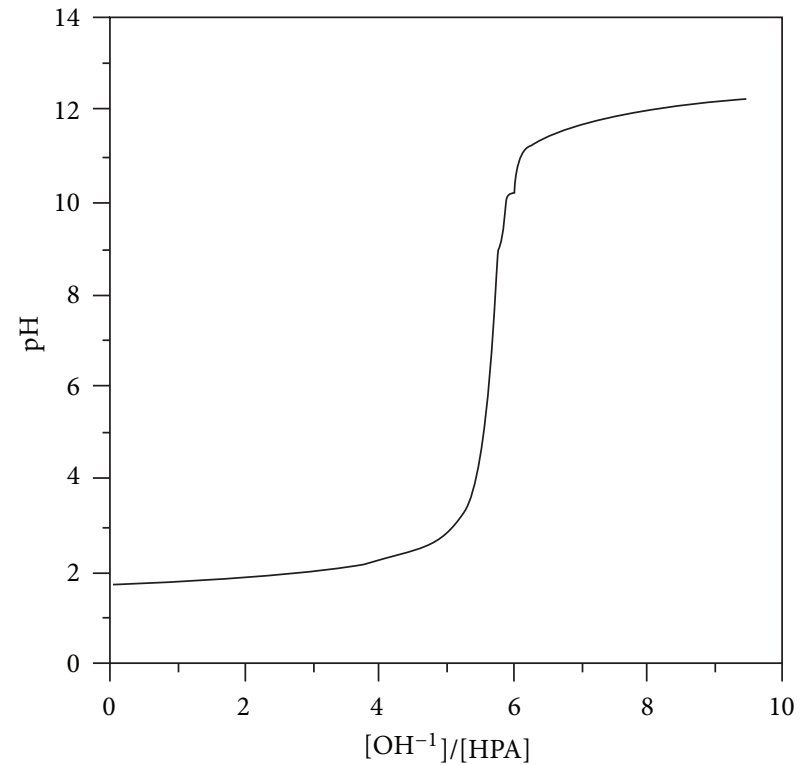

FIgURE 1: Potentiometric titration curve of $\mathrm{H}_{6} \mathrm{GeW}_{10} \mathrm{~V}_{2} \mathrm{O}_{40} \cdot 22 \mathrm{H}_{2} \mathrm{O}$.

under suction, washed with cold water $(40 \mathrm{~mL})$ and ethyl acetate $(5 \mathrm{~mL})$ to afford the pure product $\mathbf{2 a}$.

\subsection{Spectral Data of Compounds}

\section{3,3-Dimethyl-(7S, 11R)-diphenyl-2,4-dioxa-8,10-diazaspiro}

[5.5] undecane-1,5,9-trione (1a). White powder. Mp $223-225^{\circ} \mathrm{C}$ dec. IR $(\mathrm{KBr})\left(v_{\max }, \mathrm{cm}^{-1}\right): 3195$ and 3060 (NH), 1771, 1731 and $1685(\mathrm{C}=\mathrm{O}) .{ }^{1} \mathrm{H}$ NMR (DMSO, $\mathrm{Me}_{4} \mathrm{Si}$ ): $\delta_{\mathrm{H}} 0.49\left(6 \mathrm{H}, \mathrm{s}, \mathrm{CMe}_{2}\right), 5.29(2 \mathrm{H}, \mathrm{s}, 2 \mathrm{CH}), 7.20-7.37(10 \mathrm{H}, \mathrm{m}$, $\mathrm{Ar}), 7.28(2 \mathrm{H}, \mathrm{s}, 2 \mathrm{NH}) .{ }^{13} \mathrm{C}$ NMR (DMSO, $\left.\mathrm{Me}_{4} \mathrm{Si}\right): \delta_{\mathrm{C}} 27.67$ $\left(\mathrm{CMe}_{2}\right), 57.99\left(\mathrm{C}_{\text {spiro }}\right), 61.48(2 \mathrm{CH}), 105.51\left(\mathrm{CMe}_{2}\right), 127.72$, 128.71, 129.26, and 135.54 (Ar), 155.22, 159.69, $165.55(3 \mathrm{C}=\mathrm{O})$. MS (m/z, \%) $380\left(\mathrm{M}^{+}, 11\right), 322$ (7), 294 (13), 234 (12), 175 (17), 106 (100), 77 (44), 43 (56).

3,3-Dimethyl-(7S,11R)-bis(4-methylphenyl)-2,4-dioxa-8,10diazaspiro[5.5] undecane-1,5,9-trione (1b). White powder. Mp 199-200 ${ }^{\circ} \mathrm{C} \mathrm{dec}$. IR $(\mathrm{KBr})\left(v_{\max }, \mathrm{cm}^{-1}\right): 3200$ and $3060(\mathrm{NH})$, 1765, 1730 and $1686(\mathrm{C}=\mathrm{O}) .{ }^{1} \mathrm{H}$ NMR (DMSO, $\left.\mathrm{Me}_{4} \mathrm{Si}\right): \delta_{\mathrm{H}}$ $0.51\left(6 \mathrm{H}, \mathrm{s}, \mathrm{CMe}_{2}\right), 2.25\left(6 \mathrm{H}, \mathrm{s}, 2 \mathrm{CH}_{3}\right), 5.22(2 \mathrm{H}, \mathrm{s}, 2 \mathrm{CH})$, 7.07-7.2 (8H, m, Ar), 7.17 (2H, s, 2NH). ${ }^{13} \mathrm{C}$ NMR (DMSO, $\left.\mathrm{Me}_{4} \mathrm{Si}\right): \delta_{\mathrm{C}} 20.62\left(2 \mathrm{CH}_{3}\right), 27.73\left(\mathrm{CMe}_{2}\right), 57.99\left(\mathrm{C}_{\text {spiro }}\right), 61.21$ $(2 \mathrm{CH}), 105.44\left(\mathrm{CMe}_{2}\right), 127.56,129.12,132.52$ and $138.66(\mathrm{Ar})$,

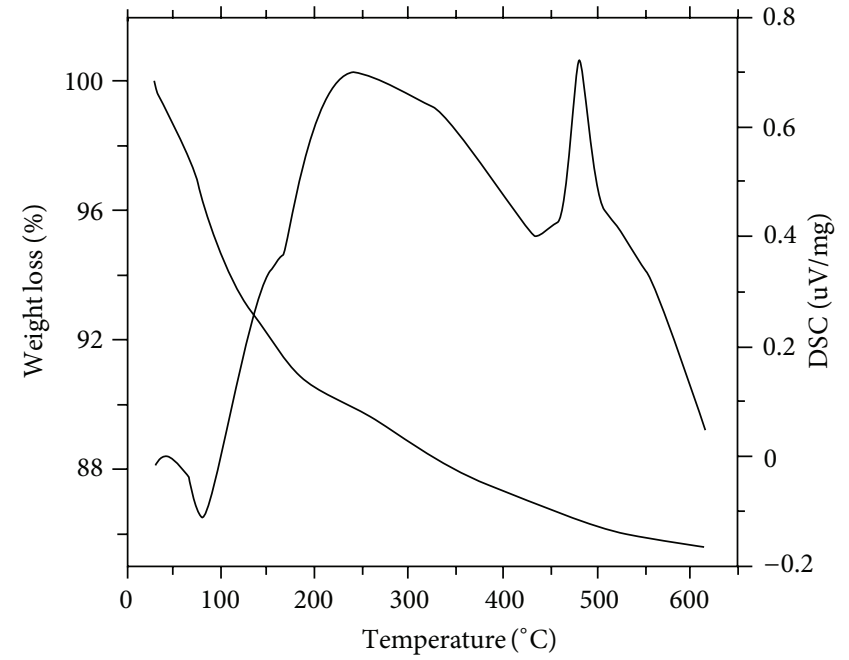

Figure 2: Thermogram of $\mathrm{H}_{6} \mathrm{GeW}_{10} \mathrm{~V}_{2} \mathrm{O}_{40} \cdot 22 \mathrm{H}_{2} \mathrm{O}$.

155.31, 159.77, $165.64(3 \mathrm{C}=\mathrm{O})$. MS $(\mathrm{m} / \mathrm{z}, \%) 408\left(\mathrm{M}^{+}, 14\right), 350$ (7), 322 (11), 189 (27), 173 (36), 120 (100), 91 (69), 75 (14), 43 (59).

3,3-Dimethyl-(7S,11R)-bis(4-chlorophenyl)-2,4-dioxa-8,10diazaspiro[5.5] undecane-1,5,9-trione (1c). White powder. Mp $204-206^{\circ} \mathrm{C} \mathrm{dec}$. IR $(\mathrm{KBr})\left(v_{\max }, \mathrm{cm}^{-1}\right): 3205$ and $3065(\mathrm{NH})$, 1770, 1731 and $1687(\mathrm{C}=\mathrm{O}) .{ }^{1} \mathrm{H} \mathrm{NMR}\left(\mathrm{DMSO}, \mathrm{Me}_{4} \mathrm{Si}\right): \delta_{\mathrm{H}} 0.60$ $\left(6 \mathrm{H}, \mathrm{s}, \mathrm{CMe}_{2}\right), 5.32(2 \mathrm{H}, \mathrm{s}, 2 \mathrm{CH}), 7.21-7.47(8 \mathrm{H}, \mathrm{m}, \mathrm{Ar}), 7.46$ $(2 \mathrm{H}, \mathrm{s}, 2 \mathrm{NH}) .{ }^{13} \mathrm{C}$ NMR (DMSO, $\left.\mathrm{Me}_{4} \mathrm{Si}\right): \delta_{\mathrm{C}} 27.81\left(\mathrm{CMe}_{2}\right)$, $57.75\left(\mathrm{C}_{\text {spiro }}\right), 60.73(2 \mathrm{CH}), 105.69\left(\mathrm{CMe}_{2}\right), 128.76,129.62$, 133.92 and $134.33(\mathrm{Ar}), 155.15,159.63$ and $165.32(3 \mathrm{C}=\mathrm{O})$. MS $(\mathrm{m} / \mathrm{z}, \%) 449\left(\mathrm{M}^{+}, 16\right), 390(7), 209(22), 173(36), 166(57), 140$ (98), 75 (14), 43 (100).

3,3-Dimethyl-(7S,11r)-bis(4-fluorophenyl)-2,4-dioxa-8,10-diazaspiro[5.5] undecane-1,5,9-trione (1d). White powder. Mp $216-218^{\circ} \mathrm{C}$ dec. IR $(\mathrm{KBr})\left(v_{\max }, \mathrm{cm}^{-1}\right): 3205$ and $3065(\mathrm{NH})$, 1770,1725 and $1680(\mathrm{C}=\mathrm{O}) .{ }^{1} \mathrm{H} \mathrm{NMR}$ (DMSO, $\left.\mathrm{Me}_{4} \mathrm{Si}\right): \delta_{\mathrm{H}} 0.59$ $\left(6 \mathrm{H}, \mathrm{s}, \mathrm{CMe}_{2}\right), 5.32(2 \mathrm{H}, \mathrm{s}, 2 \mathrm{CH}), 7.24-7.26(8 \mathrm{H}, \mathrm{m}, \mathrm{Ar}), 7.47$ $(2 \mathrm{H}, \mathrm{s}, 2 \mathrm{NH}) .{ }^{13} \mathrm{C}$ NMR (DMSO, $\left.\mathrm{Me}_{4} \mathrm{Si}\right): \delta_{\mathrm{C}} 27.80\left(\mathrm{CMe}_{2}\right)$, $58.03\left(\mathrm{C}_{\text {spiro }}\right), 60.69(2 \mathrm{CH}), 105.62\left(\mathrm{CMe}_{2}\right), 115.65,129.92$, 131.64 and $155.27(\mathrm{Ar}), 159.79,163.45$ and $165.48(3 \mathrm{C}=\mathrm{O})$. MS $(\mathrm{m} / z, \%) 417\left(\mathrm{M}^{+}+1,136\right), 358(12), 316(9), 193(26), 149$ (68), 124 (90), 75 (34), 43 (100). 
(7S,11R)-Diphenyl-2,4,8,10-tetraazaspiro[5.5] undecane-1,3,5, 9-tetraone (1e). White powder. Mp $240-242^{\circ} \mathrm{C}$ dec. IR (KBr) $\left(\nu_{\max }, \mathrm{cm}^{-1}\right): 3240$ and $3065(\mathrm{NH}), 1729$ and $1695(\mathrm{C}=\mathrm{O}) .{ }^{1} \mathrm{H}$ NMR (DMSO, $\left.\mathrm{Me}_{4} \mathrm{Si}\right): \delta_{\mathrm{H}} 5.21(2 \mathrm{H}, \mathrm{s}, 2 \mathrm{CH}), 7.17-7.31(10 \mathrm{H}$, $\mathrm{m}, \mathrm{Ar}), 7.31(2 \mathrm{H}, \mathrm{s}, 2 \mathrm{NH}), 11.01$ and $11.39(2 \mathrm{H}, 2 \mathrm{~s}, \mathrm{NH}) .{ }^{13} \mathrm{C}$ $\mathrm{NMR}$ (DMSO, $\left.\mathrm{Me}_{4} \mathrm{Si}\right): \delta_{\mathrm{C}} 57.49\left(\mathrm{C}_{\text {spiro }}\right), 61.59(2 \mathrm{CH}), 127.81$, 128.91, 129.36 and 136.12 (Ar), 149.11, 156.05, 165.88 and 170.31 $(4 \mathrm{C}=\mathrm{O}) . \mathrm{MS}(\mathrm{m} / z, \%) 364\left(\mathrm{M}^{+}, 5\right), 304(10), 215(95), 104(100)$, 77 (96), 51 (98).

(7S,11R)-bis(4-Methylphenyl)-2,4,8,10-tetraazaspiro[5.5]undecane-1,3,5,9-tetraone (lf). White powder. Mp $246-248^{\circ} \mathrm{C}$ dec. IR $(\mathrm{KBr})\left(\nu_{\max }, \mathrm{cm}^{-1}\right): 3235$ and $2975(\mathrm{NH}), 1724$ and $1692(\mathrm{C}=\mathrm{O}) .{ }^{1} \mathrm{H}$ NMR (DMSO, $\left.\mathrm{Me}_{4} \mathrm{Si}\right): \delta_{\mathrm{H}} 2.23\left(6 \mathrm{H}, \mathrm{s}, 2 \mathrm{CH}_{3}\right)$, $5.14(2 \mathrm{H}, \mathrm{s}, 2 \mathrm{CH}), 7.03-7.11(8 \mathrm{H}, \mathrm{m}, \mathrm{Ar}), 7.01(2 \mathrm{H}, \mathrm{s}, 2 \mathrm{NH})$, 10.97 and $11.33(2 \mathrm{H}, 2 \mathrm{~s}, \mathrm{NH}) .{ }^{13} \mathrm{C} \mathrm{NMR}$ (DMSO, $\left.\mathrm{Me}_{4} \mathrm{Si}\right): \delta_{\mathrm{C}}$ $20.66\left(2 \mathrm{CH}_{3}\right), 57.02\left(\mathrm{C}_{\text {spiro }}\right), 60.91(2 \mathrm{CH}), 127.21,128.98,132.66$ and 138.11 (Ar), 148.75, 155.66, 165.51 and $169.94(\mathrm{C}=\mathrm{O})$. MS $(\mathrm{m} / z, \%) 364\left(\mathrm{M}^{+}-\mathrm{CO}, 7\right), 338$ (25), 277 (31), 215 (100), 105 (87), 91 (23), 77 (39), 51 (45).

(7S,11R)-bis(4-Chlorophenyl)-2,4,8,10-tetraazaspiro[5.5] undecane-1,3,5,9-tetraone (1g). Cream powder. Mp 291-293 ${ }^{\circ} \mathrm{C}$ dec. IR $(\mathrm{KBr})\left(\nu_{\max }, \mathrm{cm}^{-1}\right): 3146$ and $3065(\mathrm{NH}), 1735$ and $1708(\mathrm{C}=\mathrm{O}) .{ }^{1} \mathrm{H}$ NMR (DMSO, $\left.\mathrm{Me}_{4} \mathrm{Si}\right): \delta_{\mathrm{H}} 5.21(2 \mathrm{H}, \mathrm{s}, 2 \mathrm{CH})$, $7.15-7.41(8 \mathrm{H}, \mathrm{m}, \mathrm{Ar}), 7.20(2 \mathrm{H}, \mathrm{s}, 2 \mathrm{NH}), 11.14$ and $11.51(2 \mathrm{H}$, 2s, NH). ${ }^{13} \mathrm{C}$ NMR (DMSO, $\left.\mathrm{Me}_{4} \mathrm{Si}\right): \delta_{\mathrm{C}} 56.82\left(\mathrm{C}_{\text {spiro }}\right), 60.33$ (2CH), 128.48, 129.23, 133.47 and 134.50 (Ar), 148.58, 155.42, 165.18 and $169.47(\mathrm{C}=\mathrm{O})$. MS $(\mathrm{m} / z, \%) 432\left(\mathrm{M}^{+}-1,10\right), 400$ (35), 372 (26), 249 (78), 215 (56), 138 (100), 75 (39), 51 (69).

(7S,11R)-bis(4-Fluorophenyl)-2,4,8,10-tetraazaspiro[5.5]undecane-1,3,5,9-tetraone (1h). White powder. Mp $213-215^{\circ} \mathrm{C}$ dec. IR $(\mathrm{KBr})\left(\nu_{\max }, \mathrm{cm}^{-1}\right): 3195$ and $3070(\mathrm{NH}), 1757,1694$ $(\mathrm{C}=\mathrm{O}) .{ }^{1} \mathrm{H}$ NMR (DMSO, $\left.\mathrm{Me}_{4} \mathrm{Si}\right): \delta_{\mathrm{H}} 5.21(2 \mathrm{H}, \mathrm{s}, 2 \mathrm{CH}), 7.11-$ $7.22(8 \mathrm{H}$, bs, Ar), $7.29(2 \mathrm{H}, \mathrm{s}, 2 \mathrm{NH}), 11.15$ and $11.49(2 \mathrm{H}$, 2s, NH). ${ }^{13} \mathrm{C}$ NMR (DMSO, $\left.\mathrm{Me}_{4} \mathrm{Si}\right): \delta_{\mathrm{C}} 57.05\left(\mathrm{C}_{\text {spiro }}\right), 60.28$ $(2 \mathrm{CH}), 115.27,129.43,131.73$ and 150.19 (Ar), 155.47, 161.20, 165.35 and $169.62(\mathrm{C}=\mathrm{O})$. MS $(\mathrm{m} / z, \%) 400\left(\mathrm{M}^{+}, 10\right), 350(25)$, 233 (100), 190 (56), 122 (98), 95 (73), 75 (69), 51 (69).

2,4-Dimethyl-(7S,11R)-diphenyl-2,4,8,10-tetraazaspiro[5.5] undecane-1,3,5,9-tetraone (1i). White powder. Mp 232-234 ${ }^{\circ} \mathrm{C}$ dec. IR $(\mathrm{KBr})\left(v_{\max }, \mathrm{cm}^{-1}\right): 3180$ and $3060(\mathrm{NH}), 1739$ and $1685(\mathrm{C}=\mathrm{O}) .{ }^{1} \mathrm{H}$ NMR (DMSO, $\mathrm{Me}_{4} \mathrm{Si}$ ): $\delta_{\mathrm{H}} 2.68$ and 2.85 $(6 \mathrm{H}, \mathrm{s}, 2 \mathrm{NMe}), 5.28$ (2H, s, 2CH), 7.08-7.28 (10H, m, Ar), 7.18 $(2 \mathrm{H}, \mathrm{s}, 2 \mathrm{NH}) .{ }^{13} \mathrm{C}$ NMR (DMSO, $\left.\mathrm{Me}_{4} \mathrm{Si}\right): \delta_{\mathrm{C}} 27.87$ and 28.71 (2NMe), $58.83\left(\mathrm{C}_{\text {spiro }}\right), 62.04(2 \mathrm{CH}), 127.43,128.84,129.49$, and 135.93 (Ar), 149.44, 155.87, 163.67 and $168.27(4 \mathrm{C}=\mathrm{O})$. MS $(\mathrm{m} / z, \%) 392\left(\mathrm{M}^{+}, 17\right), 260$ (13), 243 (31), $186(18), 106$ (100), 77 (39), 51 (33).

2,4-Dimethyl-(7S,11R)-bis(4-methylphenyl)-2,4,8,10-tetraazaspiro[5.5] undecane-1,3,5,9-tetraone (1j). White powder. Mp $228-230^{\circ} \mathrm{C}$ dec. IR $(\mathrm{KBr})\left(\nu_{\max }, \mathrm{cm}^{-1}\right): 3195$ and $3055(\mathrm{NH})$, 1738 and $1686(\mathrm{C}=\mathrm{O}) .{ }^{1} \mathrm{H}$ NMR (DMSO, $\left.\mathrm{Me}_{4} \mathrm{Si}\right): \delta_{\mathrm{H}} 2.21(6 \mathrm{H}$, s, $\left.2 \mathrm{CH}_{3}\right), 2.71$ and $2.85(6 \mathrm{H}, \mathrm{s}, 2 \mathrm{NMe}), 5.22(2 \mathrm{H}, \mathrm{s}, 2 \mathrm{CH})$, 6.97-7.09 (8H, m, Ar), 7.08 (2H, s, 2NH). ${ }^{13} \mathrm{C}$ NMR (DMSO, $\left.\mathrm{Me}_{4} \mathrm{Si}\right): \delta_{\mathrm{C}} 20.64\left(2 \mathrm{CH}_{3}\right), 27.42$ and $28.72(2 \mathrm{NMe}), 58.28$
$\left(\mathrm{C}_{\text {spiro }}\right), 61.36(2 \mathrm{CH}), 126.84,128.86,132.51$, and 138.21 (Ar), $149.40,155.35,163.28$ and $167.83(4 \mathrm{C}=\mathrm{O})$. MS $(\mathrm{m} / z, \%) 420$ $\left(\mathrm{M}^{+}, 10\right), 360$ (6), 274 (28), 257 (31), 186 (13), 120 (100), 106 (11), 91 (23), 77 (9).

2,4-Dimethyl-(7S,11R)-bis(4-chlorophenyl)-2,4,8,10-tetraazaspiro[5.5] undecane-1,3,5,9-tetraone (1k). White powder. Mp 271-273 ${ }^{\circ} \mathrm{C}$ dec. IR $(\mathrm{KBr})\left(\nu_{\max }, \mathrm{cm}^{-1}\right): 3195$ and $3060(\mathrm{NH})$, 1744 and $1659(\mathrm{C}=\mathrm{O}) .{ }^{1} \mathrm{H}$ NMR (DMSO, $\left.\mathrm{Me}_{4} \mathrm{Si}\right): \delta_{\mathrm{H}} 2.74$ and $2.87(6 \mathrm{H}, \mathrm{s}, 2 \mathrm{NMe}), 5.30(2 \mathrm{H}, \mathrm{s}, 2 \mathrm{CH}), 7.10-7.38(8 \mathrm{H}, \mathrm{m}$, Ar), $7.25(2 \mathrm{H}, \mathrm{s}, 2 \mathrm{NH}) .{ }^{13} \mathrm{C} \mathrm{NMR}$ (DMSO, $\left.\mathrm{Me}_{4} \mathrm{Si}\right): \delta_{\mathrm{C}} 27.53$ and $28.34(2 \mathrm{NMe}), 56.67\left(\mathrm{C}_{\text {spiro }}\right), 60.82(2 \mathrm{CH}), 128.39,128.97$, 129.36, and $133.46(\mathrm{Ar}), 155.14,156.72,159.30$ and 162.98 $(4 \mathrm{C}=\mathrm{O})$. MS $(\mathrm{m} / z, \%) 460\left(\mathrm{M}^{+}, 14\right), 400(16), 321(14), 294$ (23), 277 (89), 220 (31), 140 (100), 75 (34).

2,4-Dimethyl-(7S,11R)-bis(4-fluorophenyl)-2,4,8,10-tetraazaspiro[5.5] undecane-1,3,5,9-tetraone (1l). White powder. $\mathrm{Mp}$ $244-246^{\circ} \mathrm{C}$ dec. IR $(\mathrm{KBr})\left(\nu_{\max }, \mathrm{cm}^{-1}\right): 3190$ and $3065(\mathrm{NH})$, $1740,1656(\mathrm{C}=\mathrm{O}) .{ }^{1} \mathrm{H}$ NMR (DMSO, $\left.\mathrm{Me}_{4} \mathrm{Si}\right): \delta_{\mathrm{H}} 2.75$ and 2.87 (6H, s, 2NMe), 5.30 (2H, s, 2CH), 7.13-7.15 (8H, m, Ar), 7.26 $(2 \mathrm{H}, \mathrm{s}, 2 \mathrm{NH}) .{ }^{13} \mathrm{C} \mathrm{NMR}\left(\mathrm{DMSO}, \mathrm{Me}_{4} \mathrm{Si}\right): \delta_{\mathrm{C}} 27.47$ and 28.27 $(2 \mathrm{NMe}), 58.28\left(\mathrm{C}_{\text {spiro }}\right), 60.76(2 \mathrm{CH}), 115.21,129.20,131.60$, and 148.93 (Ar), 155.24, 161.16, 163.12 and $167.53(4 \mathrm{C}=\mathrm{O})$. MS $(\mathrm{m} / \mathrm{z}$, \%) $428\left(\mathrm{M}^{+}, 10\right), 385$ (6), 305 (17), 278 (33), 261 (69), 204 (31), 124 (100), 95 (35), 75 (34).

4,8-Diphenyloctahydro-1H-pyrimido[5,4-i]quinazoline-2,10 $(3 \mathrm{H}, 11 \mathrm{H})$-dione $(2 a)$. Mp 327-329 $\mathrm{C} ;{ }^{1} \mathrm{H}$ NMR (DMSO- $\left.d_{6}\right)$ : $\delta 7.40-7.19(\mathrm{~m}, 10 \mathrm{H}), 7.08(\mathrm{~s}, 1 \mathrm{H}), 6.97(\mathrm{~s}, 1 \mathrm{H}), 6.62(\mathrm{~s}, 1 \mathrm{H})$, $6.39(\mathrm{~s}, 1 \mathrm{H}), 4.50(\mathrm{~d}, 1 \mathrm{H}), 4.82(\mathrm{~d}, 1 \mathrm{H}), 2.02(\mathrm{~m}, 2 \mathrm{H}), 1.38$ $(\mathrm{m}, 2 \mathrm{H}), 1.24(\mathrm{~m}, 2 \mathrm{H}), 0.82(\mathrm{t}, 2 \mathrm{H}) ;{ }^{13} \mathrm{C}-\mathrm{NMR}$ (DMSO- $d_{6}$ ) $\delta: 155.9,140.5,128.1,128.6,126.0,63.7,50.2$, 49.1, 17.8; ESI-MS $377(\mathrm{M}+\mathrm{H}) ; \mathrm{C}_{22} \mathrm{H}_{24} \mathrm{~N}_{4} \mathrm{O}_{2}$; (376.45); Calcd. C, 70.19; H, 6.43; N, 14.88; O, 8.50. Found. C, 70.03; H, 6.21; N, 14.45; O, 8.23.

4,8-bis(2-Chlorophenyl)octahydro-1H-pyrimido[5,4-i]quinazoline-2,10(3H,11H)-dione $(2 d)$. Mp $321-323^{\circ} \mathrm{C} ;{ }^{1} \mathrm{H}$ NMR $\left(\mathrm{DMSO}-d_{6}\right): \delta 7.42(\mathrm{~s}, 1 \mathrm{H}), 7.35-7.10(\mathrm{~m}, 9 \mathrm{H}), 6.75(\mathrm{~s}, 1 \mathrm{H}), 5.32$ (s, 1H), $5.32(\mathrm{~s}, 1 \mathrm{H}), 3.91(\mathrm{~m}, 3 \mathrm{H}), 3.69(\mathrm{~m}, 3 \mathrm{H}), 2.30(\mathrm{~m}, 2 \mathrm{H})$, $2.01(\mathrm{~m}, 1 \mathrm{H}), 1.84(\mathrm{~m}, 1 \mathrm{H}), 1.32(\mathrm{~m}, 1 \mathrm{H}), 1.19(\mathrm{~m}, 1 \mathrm{H}), 0.89$ $(\mathrm{m}, 1 \mathrm{H}) ;{ }^{13} \mathrm{C}-\mathrm{NMR}\left(\mathrm{DMSO}-d_{6}\right) \delta: 155.9,140.5,133.4,129.5$, 128.6, 127.4, 63.7, 48.6, 45.1, 23.6, 17.8; ESI-MS $445(\mathrm{M}+\mathrm{H})$; $\mathrm{C}_{22} \mathrm{H}_{22} \mathrm{Cl}_{2} \mathrm{~N}_{4} \mathrm{O}_{2}$ (445.34); Calcd. C, 59.33; H, 4.98; Cl, 15.92; N, 12.58; O, 7.19. Found. C, 59.12; H, 4.56; Cl, 15.74; N, 12.28; $\mathrm{O}, 7.02$.

\section{Results and Discussion}

The reaction of cyclic $\beta$-ketoesters [44] and $\beta$-diamides, Meldrum's acid, or barbituric acid derivatives with 1 equivalent of urea and 2 equivalents of aldehydes gives a family of $\sigma$ symmetric spiroheterobicyclic compounds in good yields in the presence of $\mathrm{H}_{6} \mathrm{GeW}_{10} \mathrm{~V}_{2} \mathrm{O}_{40} \cdot 22 \mathrm{H}_{2} \mathrm{O}$ as a catalyst under solvent-free conditions at $80^{\circ} \mathrm{C}$ (Scheme 1 and Table 2).

To explore the scope and limitations of this reaction further, we have extended it to various para-substituted benzald- 
TABLe 2: $\mathrm{H}_{6} \mathrm{GeW}_{10} \mathrm{~V}_{2} \mathrm{O}_{40} \cdot 22 \mathrm{H}_{2} \mathrm{O}$ catalyzed synthesis of spiroheterobicyclic rings $\mathbf{1}(\mathbf{a}-\mathbf{l})$.

\begin{tabular}{lccccc}
\hline Entry & $\mathrm{X}-\mathrm{Z}-\mathrm{X}$ & $\mathrm{G}$ & Product & Yield (\%) & M.P. $\left({ }^{\circ} \mathrm{C}\right)$ \\
\hline 1 & $\mathrm{O}-\mathrm{C}(\mathrm{Me})_{2}-\mathrm{O}$ & $\mathrm{H}$ & $\mathbf{l a}$ & 80 & $223-225$ \\
2 & $\mathrm{O}-\mathrm{C}(\mathrm{Me})_{2}-\mathrm{O}$ & $\mathrm{Me}$ & $\mathbf{l b}$ & 68 & $199-200$ \\
3 & $\mathrm{O}-\mathrm{C}(\mathrm{Me})_{2}-\mathrm{O}$ & $\mathrm{Cl}$ & $\mathbf{1 c}$ & 66 & $204-206$ \\
4 & $\mathrm{O}-\mathrm{C}(\mathrm{Me})_{2}-\mathrm{O}$ & $\mathrm{F}$ & $\mathbf{1 d}$ & 67 & $216-218$ \\
5 & $\mathrm{HN}-\mathrm{CO}-\mathrm{NH}$ & $\mathrm{H}$ & $\mathbf{l e}$ & 87 & $240-242$ \\
6 & $\mathrm{Me}$ & $\mathbf{l f}$ & 84 & $246-248$ \\
7 & $\mathrm{HN}-\mathrm{CO}-\mathrm{NH}$ & $\mathrm{Cl}$ & $\mathbf{1 g}$ & 82 & $291-293$ \\
8 & $\mathrm{HN}-\mathrm{CO}-\mathrm{NH}$ & $\mathrm{F}$ & $\mathbf{1 h}$ & 77 & $213-215$ \\
9 & $\mathrm{HN}-\mathrm{CO}-\mathrm{NH}$ & $\mathrm{H}$ & $\mathbf{1 i}$ & 83 & $232-234$ \\
10 & $\mathrm{MeN}-\mathrm{CO}-\mathrm{NMe}$ & $\mathrm{Me}$ & $\mathbf{1 k}$ & 85 & $228-230$ \\
11 & $\mathrm{MeN}-\mathrm{CO}-\mathrm{NMe}$ & $\mathrm{Cl}$ & $\mathrm{il}$ & 77 & $271-273$ \\
12 & $\mathrm{MeN}-\mathrm{CO}-\mathrm{NMe}$ & $\mathrm{F}$ & & 75 & $244-246$ \\
\hline
\end{tabular}

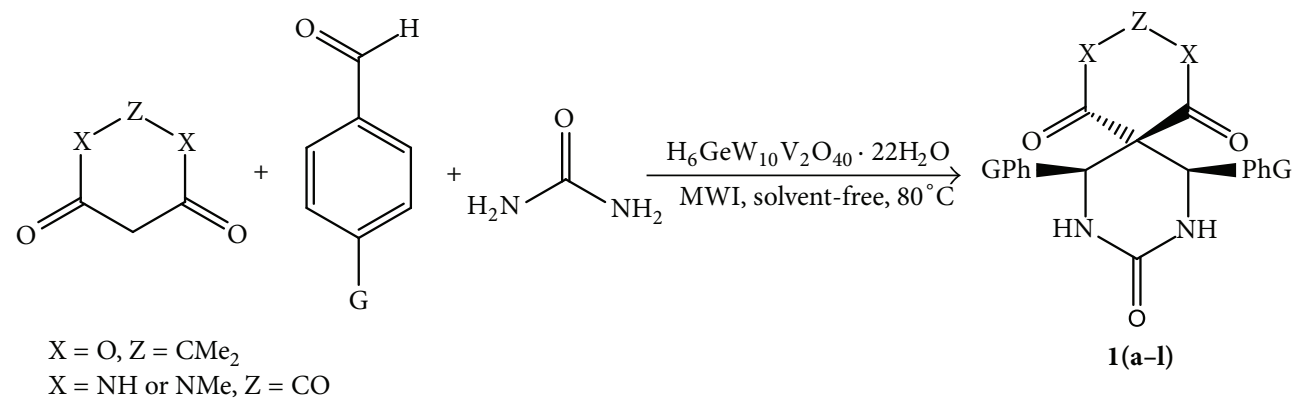

SCHEME 1


2(a-d)

SCHEME 2

ehydes in the presence of Meldrum's acid and barbituric acid (Scheme 1). We have found that the reaction proceeds very efficiently with benzaldehyde and electron withdrawing parasubstituted benzaldehydes, but it proceeded only up to Knoevenagel adducts, when electron releasing para-substituted benzaldehydes were used $\left(\mathrm{X}=\mathrm{OMe}, \mathrm{NMe}_{2}\right)$.

This investigation has been extended to cyclic ketones like cyclohexanone (Scheme 2). The products formed $\mathbf{2}(\mathbf{a}-\mathbf{d})$ are listed in Table 3.

It was shown that no desirable product could be detected when a mixture react in the absence of $\mathrm{H}_{6} \mathrm{GeW}_{10} \mathrm{~V}_{2} \mathrm{O}_{40}$. $22 \mathrm{H}_{2} \mathrm{O}$, which indicated that the catalyst should be necessary. Then the model reaction to synthesize la by the reaction of Meldrum's acid, benzaldehyde, and urea was investigated with different amounts of $\mathrm{H}_{6} \mathrm{GeW}_{10} \mathrm{~V}_{2} \mathrm{O}_{40} \cdot 22 \mathrm{H}_{2} \mathrm{O}(0-$ $5 \mathrm{~mol} \%$ ). Yields of the reaction in different conditions were shown in Table 4.

We found that most of the Lewis acids could promote the reaction, but the yields were not so high. In comparison with other catalysts, the use of $3 \mathrm{~mol} \%$ of $\mathrm{H}_{6} \mathrm{GeW}_{10} \mathrm{~V}_{2} \mathrm{O}_{40} \cdot 22 \mathrm{H}_{2} \mathrm{O}$ could make the yield $80 \%$ under the microwave power of $600 \mathrm{~W}$ and the irradiation time of $7 \mathrm{~min}$. It could be seen that $3 \mathrm{~mol} \%$ of $\mathrm{H}_{6} \mathrm{GeW}_{10} \mathrm{~V}_{2} \mathrm{O}_{40} \cdot 22 \mathrm{H}_{2} \mathrm{O}$ gave the best result of this reaction, although other factors could not yet be optimized.

Based on the above optimized results, that is, $3 \mathrm{~mol} \%$ amount of $\mathrm{H}_{6} \mathrm{GeW}_{10} \mathrm{~V}_{2} \mathrm{O}_{40} \cdot 22 \mathrm{H}_{2} \mathrm{O}$ as a catalyst, we further examined the effects of the microwave power and the irradiation time on the same model reaction to afford la, as shown 
TABLE 3: $\mathrm{H}_{6} \mathrm{GeW}_{10} \mathrm{~V}_{2} \mathrm{O}_{40} \cdot 22 \mathrm{H}_{2} \mathrm{O}$ catalyzed reaction of cyclohexanone, aldehyde, and urea.

\begin{tabular}{lcccc}
\hline Entry & $\mathrm{R}$ & Products $^{\mathrm{a}}$ & Yield $^{\mathrm{b}}(\%)$ & $\mathrm{M} . \mathrm{P}\left({ }^{\circ} \mathrm{C}\right)$ \\
\hline 1 & $\mathrm{C}_{6} \mathrm{H}_{5}$ & 2a & 87 & $327-329$ \\
2 & $4-\left(\mathrm{NO}_{2}\right) \mathrm{C}_{6} \mathrm{H}_{4}$ & 2b & 79 & $341-343$ \\
3 & $4-\left(\mathrm{CH}_{3}\right) \mathrm{C}_{6} \mathrm{H}_{4}$ & 2c & 83 & $348-351$ \\
4 & $2-(\mathrm{Cl})-\mathrm{C}_{6} \mathrm{H}_{4}$ & 2d & 82 & $321-323$ \\
\hline
\end{tabular}

${ }^{a}$ Reaction conditions: cyclohexanone $(1.0 \mathrm{mmol})$, aldehyde $(2.0 \mathrm{mmol})$, urea $(3.0 \mathrm{mmol})$, and decatungstodivanadogermanic acid $(3 \mathrm{mmol})$ irradiated at $80^{\circ} \mathrm{C}$ under solvent-free condition.

TABLE 4: Yields of the reaction in different conditions.

\begin{tabular}{lcc}
\hline $\begin{array}{l}\text { Amount of catalyst } \\
(\% \mathrm{~mol})\end{array}$ & $\begin{array}{c}\text { Reaction time } \\
(\mathrm{min}) / \text { temperature }\left({ }^{\circ} \mathrm{C}\right)\end{array}$ & Yields $(\%)$ \\
\hline 0 & $7 / 80$ & 46 \\
1 & $7 / 80$ & 52 \\
2 & $7 / 80$ & 63 \\
3 & $7 / 80$ & 80 \\
4 & $7 / 80$ & 75 \\
5 & $7 / 80$ & 73 \\
\hline
\end{tabular}

TABLE 5: Effect of the microwave power and the irradiation time on the formation of $\mathbf{l a}$.

\begin{tabular}{lccc}
\hline Entry & Time $(\min )$ & Power $(\mathrm{W})$ & Yields $(\%)$ \\
\hline 1 & 4 & 250 & 47 \\
2 & 4 & 300 & 52 \\
3 & 4 & 400 & 55 \\
4 & 4 & 500 & 58 \\
5 & 4 & 600 & 63 \\
6 & 4 & 700 & 69 \\
7 & 4 & 750 & 71 \\
8 & 4 & 800 & 74 \\
9 & 4 & 900 & 80 \\
10 & 2 & 900 & 36 \\
11 & 3 & 900 & 62 \\
12 & 5 & 900 & 88 \\
13 & 7 & 900 & 97 \\
14 & 8 & 900 & 94 \\
15 & 9 & 900 & 92 \\
\hline
\end{tabular}

Reaction conditions: benzaldehyde $(0.30 \mathrm{~g}, 2 \mathrm{mmol})$, Meldrum's acid $(0.144 \mathrm{~g}, 1 \mathrm{mmol})$, urea $(0.06 \mathrm{~g}, 1 \mathrm{mmol})$, and decatungstodivanadogermanic acid $(0.03 \mathrm{~g}, 3 \mathrm{mmol})$ in microwave irradiation at $80^{\circ} \mathrm{C}$ under solvent-free condition.

in Scheme 1. The results are listed in Table 5. It could be found that with the increase of the microwave power from $250 \mathrm{~W}$ to $900 \mathrm{~W}$, the yield of 1a showed a linear increase from $47 \%$ to $80 \%$ when the irradiation time was $4 \mathrm{~min}$. However, with the microwave power of $900 \mathrm{~W}$, when we increased the microwave irradiation time, the yield of 1a increased first, but a slight decrease was observed for more than $7 \mathrm{~min}$. So the optimized microwave power and the irradiation time were $900 \mathrm{~W}$ and $7 \mathrm{~min}$, respectively.
TABLE 6: Comparison of the results of the present work with those of the earlier works.

\begin{tabular}{lcccc}
\hline Catalyst & Conditions & Yield (\%) & Time & Reference \\
\hline $\mathrm{NBS} / \mathrm{AIBN}$ & Solvent-free $/ 80^{\circ} \mathrm{C}$ & $72-74$ & $4 \mathrm{~h}$ & {$[6]$} \\
$\mathrm{AlCl}_{3}$ & Ethanol/Reflux & $82-84$ & $5 \mathrm{~h}$ & {$[7]$} \\
$\mathrm{H}_{6} \mathrm{GeW}_{10} \mathrm{~V}_{2} \mathrm{O}_{40}$ & MWI/Solvent free & $87-90$ & $6-7$ min & This work \\
$22 \mathrm{H}_{2} \mathrm{O}$ & & & & \\
\hline
\end{tabular}

TABLE 7: Reusability of the catalyst for the synthesis of 3,3-dimethyl(7S, 11R)-diphenyl-2,4-dioxa-8,10-diazaspiro[5.5] undecane-1,5,9trione $e^{\mathrm{a}}$.

\begin{tabular}{lccccc}
\hline Cycle & 0 & 1st & 2nd & 3rd & 4th \\
\hline Time (min) & 7 & 7 & 8 & 9 & 9 \\
Yield $(\%)^{\mathrm{b}}$ & 80 & 78 & 76 & 73 & 71 \\
\hline
\end{tabular}

${ }^{a}$ Reaction conditions: benzaldehyde $(0.30 \mathrm{~g}, 2 \mathrm{mmol})$, Meldrum's acid $(0.144 \mathrm{~g}, 1 \mathrm{mmol})$, urea $(0.06 \mathrm{~g}, 1 \mathrm{mmol})$, and decatungstodivanadogermanic acid $(0.03 \mathrm{~g}, 3 \mathrm{mmol})$ in microwave irradiation at $80^{\circ} \mathrm{C}$ under solvent-free condition.

${ }^{\mathrm{b}}$ Isolated yields.

In order to show the merit of the present work in terms of time, yield, and reaction conditions in comparison to the earlier reported works, the results of the present study were compared with those of the earlier studies in Table 6. As it can be seen from Table 6 , the present method is simpler, more efficient for the synthesis of dihydropyrimidinone derivatives.

In order to confirm the reusability of $\mathrm{H}_{6} \mathrm{GeW}_{10} \mathrm{~V}_{2} \mathrm{O}_{40}$. $22 \mathrm{H}_{2} \mathrm{O}$ catalyst, after the completion of the reaction it was separated from the reaction mixture and washed with ethyl acetate. The recovered catalyst was found to be reusable for four cycles without significant loss in activity (Table 7). At the same time the concentrations of Wand $\mathrm{V}$ in the filtrate were determined to be less than $1 \%$ by ICP-AES. On the other hand, the IR and UV-Vis spectra of the recovered catalyst were identical with fresh catalyst. All these findings confirm that the leaching of the catalyst did not take place under the reaction conditions.

\section{Conclusion}

In conclusion we have investigated the application of a V-containing HPA as a green and recyclable heterogeneous catalyst for the synthesis spirofused heterocycles from one-pot threecomponent cyclocondensation reaction of a cyclic ketone, aldehyde, and urea in high yields under solvent-free condition in microwave irradiation. It is an efficient, mild, and green method for the synthesis of spirofused heterocycles. It is noteworthy that the catalyst can be used for subsequent cycles without appreciable loss of activity. In contrast to many other acids, the storage of this nonhygroscopic and noncorrosive solid heteropoly acid does not require special precautions; for example, it can be stored on a bench top for months without losing its catalytic activity. 


\section{Acknowledgment}

The financial support from Madhya Pradesh Council of Science \& Technology (MPCST) is highly appreciated.

\section{References}

[1] C. O. Kappe, W. M. F. Fabian, and M. A. Semones, "Conformational analysis of 4-aryl-dihydropyrimidine calcium channel modulators. A comparison of ab initio, semiempirical and X-ray crystallographic studies," Tetrahedron, vol. 53, no. 8, pp. 28032816, 1997.

[2] G. C. Rovnyak, S. D. Kimball, B. Beyer et al., "Calcium entry blockers and activators: conformational and structural determinants of dihydropyrimidine calcium channel modulators," Journal of Medicinal Chemistry, vol. 38, no. 1, pp. 119-129, 1995.

[3] B. B. Snider and Z. Shi, "Biomimetic syntheses of ( \pm )-crambines $\mathrm{A}, \mathrm{B}, \mathrm{C1}$, and C2. Revision of the structures of crambines B and C1," Journal of Organic Chemistry, vol. 58, no. 15, pp. 3828-3839, 1993.

[4] C. O. Kappe, "100 years of the Biginelli dihydropyrimidine synthesis," Tetrahedron, vol. 49, no. 32, pp. 6937-6963, 1993.

[5] J. Barluenga, M. Tomás, A. Ballesteros, and L. A. López, "1,4Cycloaddition of 1,3-diazabutadienes with enamines: an efficient route to the pyrimidine ring," Tetrahedron Letters, vol. 30, no. 34, pp. 4573-4576, 1989.

[6] S. R. Jetti, D. Verma, and S. Jain, "NBS/AIBN promoted one-pot multi component regioselective synthesis of spiro heterobicyclic rings via Biginelli-like condensation reaction," Journal of Chemical and Pharmaceutical Research, vol. 4, no. 5, pp. 2373-2379, 2012.

[7] S. R. Jetti, D. Verma, and S. Jain, "An efficient one-pot green protocol for the synthesis of 5-unsubstituted 3, 4-dihydropyrimidin-2(1H)-ones using recyclable amberlyst 15 DRY as a heterogeneous catalyst via three-component Biginelli-like reaction," ISRN Organic Chemistry, vol. 2012, Article ID 480989, 8 pages, 2012.

[8] S. Panda, K. Siva, K. Pankaj, and Leena, "Biginelli reaction: a green perspective," Current Organic Chemistry, vol. 16, no. 4, pp. 507-520, 2012.

[9] Suresh and S. J. Sandhu, "Past, present and future of the Biginelli reaction: a critical perspective," Arkivoc, vol. 1, pp. 66-133, 2012.

[10] A. Borse, P. Mahesh, P. Nilesh, and S. Rohan, "A green, expeditious, one-pot synthesis of 3, 4-dihydropyrimidin-2(1H)-ones using a mixture of phosphorus pentoxide-methanesulfonic acid at ambient temperature," ISRN Organic Chemistry, vol. 2012, Article ID 415645, 6 pages, 2012.

[11] D. Abdelmadjid, C. Louisa, B. Raouf, and C. Bertrand, "A onepot multi-component synthesis of dihydropyrimidinone/thione and dihydropyridine derivatives via Biginelli and Hantzsch condensations using $t$ - $\mathrm{BuOK}$ as a catalyst under solvent-free conditions," The Open Organic Chemistry Journal, vol. 6, pp. 1220, 2012.

[12] T. Reza, M. Behrooz, and G. Malihe, "Ammonium dihydrogen phosphate catalyst for one-pot synthesis of 3, 4-dihydropyrimidin-2(1H)-ones," Chinese Journal of Catalysis, vol. 33, no. 4-6, pp. 659-665, 2012

[13] S. Agarwal, U. Aware, A. Patil et al., "Silica-gel catalyzed facile synthesis of 3, 4-dihydropyrimidinones," Bulletin of Korean Chemical Society, vol. 33, no. 2, pp. 377-378, 2012.
[14] R. Hekmatshoar, M. Heidari, M. M. Heravi, and B. Baghernejad, "Mesoporous molecular sieve MCM-41 catalyzed onepot synthesis of 3,4-dihydro-2(1H)-pyrimidinones and -thiones under solvent-free conditions," Bulletin of the Chemical Society of Ethiopia, vol. 25, no. 2, pp. 309-313, 2011.

[15] J. A. Kumar, C. Shanmugam, and P. H. Babu, "One pot synthesis of dihydropyrimidinones catalyzed by Cyanuric chloride: an improved procedure for the Biginelli reaction," Der Pharma Chemica, vol. 3, no. 4, pp. 292-297, 2011.

[16] B. F. Mirjalili, A. Bamoniri, and A. Akbari, "One-pot synthesis of 3,4-dihydropyrimidin-2( $1 H$ )-ones (thiones) promoted by nano- $\mathrm{BF}_{3} . \mathrm{SiO}_{2}$," Journal of the Iranian Chemical Society, vol. 8 , no. 1, pp. S135-S140, 2011.

[17] M. Zeinali-Dastmalbaf, A. Davoodnia, M. M. Heravi, N. Tavakoli-Hoseini, A. Khojastehnezhad, and H. A. Zamani, "Silica gel-supported polyphosphoric acid $\left(\mathrm{PPA}-\mathrm{SiO}_{2}\right)$ catalyzed one-pot multi-component synthesis of 3, 4-dihydropyrimidin2(1H)-ones and -thiones: an efficient method for the Biginelli reaction," Bulletin of the Korean Chemical Society, vol. 32, no. 2, pp. 656-658, 2011.

[18] C. V. Reddy, M. Mahesh, P. V. K. Raju, T. R. Babu, and V. V. N. Reddy, "Zirconium(IV) chloride catalyzed one-pot synthesis of 3,4-dihydropyrimidin-2(1H)-ones," Tetrahedron Letters, vol. 43, no. 14, pp. 2657-2659, 2002.

[19] N. Y. Fu, Y. F. Yuan, Z. Cao, S. W. Wang, J. T. Wang, and C. Peppe, "Indium(III) bromide-catalyzed preparation of dihydropyrimidinones: improved protocol conditions for the Biginelli reaction," Tetrahedron, vol. 58, no. 24, pp. 4801-4807, 2002.

[20] A. Dondoni and A. Massi, "Parallel synthesis of dihydropyrimidinones using $\mathrm{Yb}(\mathrm{III})$-resin and polymer-supported scavengers under solvent-free conditions. A green chemistry approach to the Biginelli reaction," Tetrahedron Letters, vol. 42, no. 45, pp. 7975-7978, 2001.

[21] J. Peng and Y. Deng, "Ionic liquids catalyzed Biginelli reaction under solvent-free conditions," Tetrahedron Letters, vol. 42, no. 34, pp. 5917-5919, 2001.

[22] J. S. Yadav, B. V. S. Reddy, K. B. Reddy, K. S. Raj, and A. R. Prasad, "Ultrasound-accelerated synthesis of 3,4-dihydropyrimidin$2(1 H)$-ones with ceric ammonium nitrate," Journal of the Chemical Society, no. 16, pp. 1939-1941, 2001.

[23] K. A. Kumar, M. Kasthuraiah, C. S. Reddy, and C. D. Reddy, " $\mathrm{Mn}(\mathrm{OAc}) 3 \cdot 2 \mathrm{H} 2 \mathrm{O}$-mediated three-component, one-pot, condensation reaction: an efficient synthesis of 4 -aryl-substituted 3,4-dihydropyrimidin-2-ones," Tetrahedron Letters, vol. 42, no. 44, pp. 7873-7875, 2001.

[24] Y. Ma, C. Qian, L. Wang, and M. Yang, "Lanthanide triflate catalyzed biginelli reaction. One-pot synthesis of dihydropyrimidinones under solvent-free conditions," Journal of Organic Chemistry, vol. 65, no. 12, pp. 3864-3868, 2000.

[25] B. C. Ranu, A. Hajra, and U. Jana, "Indium(III) chloride-catalyzed one-pot synthesis of dihydropyrimidinones by a threecomponent coupling of 1,3-dicarbonyl compounds, aldehydes, and urea: an improved procedure for the Biginelli reaction," Journal of Organic Chemistry, vol. 65, no. 19, pp. 6270-6272, 2000.

[26] J. Lu, Y. Bai, Z. Wang, B. Yang, and H. Ma, “One-pot synthesis of 3,4-dihydropyrimidin-2(1H)-ones using lanthanum chloride as a catalyst," Tetrahedron Letters, vol. 41, no. 47, pp. 9075-9078, 2000.

[27] J. C. Bussolari and P. A. McDonnell, "A new substrate for the Biginelli cyclocondensation: direct preparation of 5-unsub- 
stituted 3,4-dihydropyrimidin-2(1H)-ones from a $\beta$-keto carboxylic acid," Journal of Organic Chemistry, vol. 65, no. 20, pp. 6777-6779, 2000.

[28] F. Bigi, S. Carloni, B. Frullanti, R. Maggi, and G. Sartori, "A revision of the biginelli reaction under solid acid catalysis. Solvent-free synthesis of dihydropyrimidines over montmorillonite KSF," Tetrahedron Letters, vol. 40, no. 17, pp. 3465-3468, 1999.

[29] C. O. Kappe, D. Kumar, and R. S. Varma, "Microwave-assisted high-speed parallel synthesis of 4-aryl-3,4-dihydropyrimidin2(1H)-ones using a solventless biginelli condensation protocol," Synthesis, no. 10, pp. 1799-1803, 1999.

[30] E. H. Hu, D. R. Sidler, and U. H. Dolling, "Unprecedented catalytic three component one-pot condensation reaction: an efficient synthesis of 5-alkoxycarbonyl-4-aryl-3,4-dihydropyrimidin- 2(1H)-ones," Journal of Organic Chemistry, vol. 63, no. 10, pp. 3454-3457, 1998.

[31] K. S. Atwal, G. C. Rovnyak, B. C. O’Reilly, and J. Schwartz, "Substituted 1,4-dihydropyrimidines. 3. Synthesis of selectively functionalized 2-hetero-1,4-dihydropyrimidines," Journal of Organic Chemistry, vol. 54, no. 25, pp. 5898-5907, 1989.

[32] V. I. Saloutin, Y. V. Burgart, O. G. Kuzueva, C. O. Kappe, and O. N. Chupakhin, "Biginelli condensations of fluorinated 3-oxo esters and 1,3-diketones," Journal of Fluorine Chemistry, vol. 103, no. 1, pp. 17-23, 2000.

[33] I. V. Kozhevnikov, "Catalysis by heteropoly acids and multicomponent polyoxometalates in liquid-phase reactions," Chemical Reviews, vol. 98, no. 1, pp. 171-198, 1998.

[34] I. V. Kozhevnikov, "Sustainable heterogeneous acid catalysis by heteropoly acids," Journal of Molecular Catalysis A, vol. 262, no. 1-2, pp. 86-92, 2007.

[35] F. Saeid and P. Somayeh, "Decatungstodivanadogermanic heteropoly acid $\left(\mathrm{H}_{6} \mathrm{GeW}_{10} \mathrm{~V}_{2} \mathrm{O}_{40} \cdot 22 \mathrm{H}_{2} \mathrm{O}\right)$ : a novel, green and reusable catalyst for efficient acetylation of alcohols and phenols under solvent-free conditions," European Journal of Chemistry, vol. 1, no. 4, pp. 335-340, 2010.

[36] Y. Zhu, Y. Pan, and S. Huang, "Trimethylsilyl chloride: a facile and efficient reagent for one-pot synthesis of 3,4-dihydropyrimidin-2(1H)-ones," Synthetic Communications, vol. 34, no. 17, pp. 3167-3174, 2004.

[37] S. R. Jetti, D. Verma, and S. Jain, "Carbon-based solid acid as an efficient and reusable catalyst for the synthesis of 4, 6-diarylpyrimidin-2(1H)-ones under solvent-free conditions," Der Chemica Sinica, vol. 3, no. 3, pp. 636-640, 2012.

[38] J. S. Rao, G. N. Babu, P. Pradeep, B. Anjna, T. Kadre, and J. Shubha, "Amberlyst 15 DRY resin: a green and recyclable catalyst for facile and efficient one-pot synthesis of 3, 4-dihydropyrimidin-2(1H)-ones," Der Pharma Chemica, vol. 4, no. 1, pp. 417-427, 2012.

[39] T. Kadre, S. R. Jetti, A. Bhatewara, P. Paliwal, and S. Jain, "Green protocol for the synthesis of 3, 4-Dihydropyrimidin$2(1 H)$-ones/thiones using TBAB as a catalyst and solvent free condition under microwave irradiation," Archives of Applied Science Research, vol. 4, no. 2, pp. 988-993, 2012.

[40] P. Pradeep, J. S. Rao, and J. Shubha, "DABCO promoted multicomponent one-pot synthesis of xanthene derivatives," Research Journal of Chemical Sciences, vol. 2, no. 8, pp. 21-25, 2012.

[41] Q. Wu, Q. Chen, X. Cai, J. Wang, and J. Zhang, "Preparation and conductivity of solid high-proton conductor silica gel containing 80 wt.\% decatungstodivanadogermanic acid," Materials Letters, vol. 61, no. 3, pp. 663-665, 2007.
[42] Q. Y. Wu, S. K. Wang, D. N. Li, and X. F. Xie, "Preparation and characterization of decatungstomolybdoniobogermanic heteropoly acid H5[GeW10MoNbO40] · 20H2O," Inorganic Chemistry Communications, vol. 5, no. 5, pp. 308-311, 2002.

[43] U. B. Mioe, M. R. Todorovic, S. M. Uskokovic-Markovic et al., "Structure and proton conductivity in a magnesium salt of 12tungstophosphoric acid," Solid State Ionics, vol. 162-163, no. EX1-EX-8, pp. 217-223, 2003.

[44] G. Byk, H. E. Gottlieb, J. Herscovici, and F. Mirkin, "New regioselective multicomponent reaction: one pot synthesis of spiro heterobicyclic aliphatic rings," Journal of Combinatorial Chemistry, vol. 2, no. 6, pp. 732-735, 2000. 

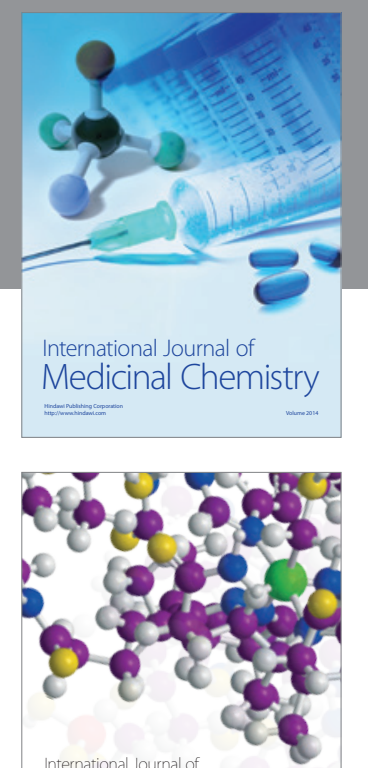

\section{Carbohydrate} Chemistry

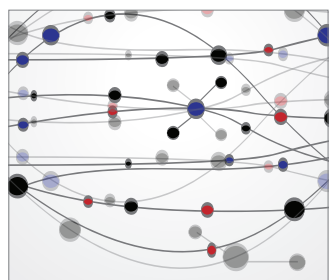

The Scientific World Journal
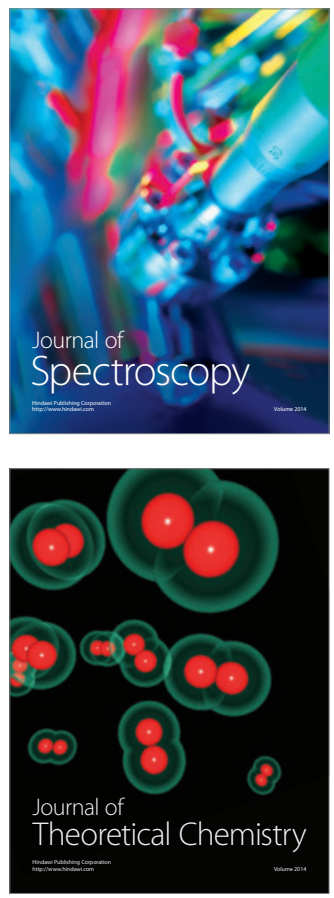
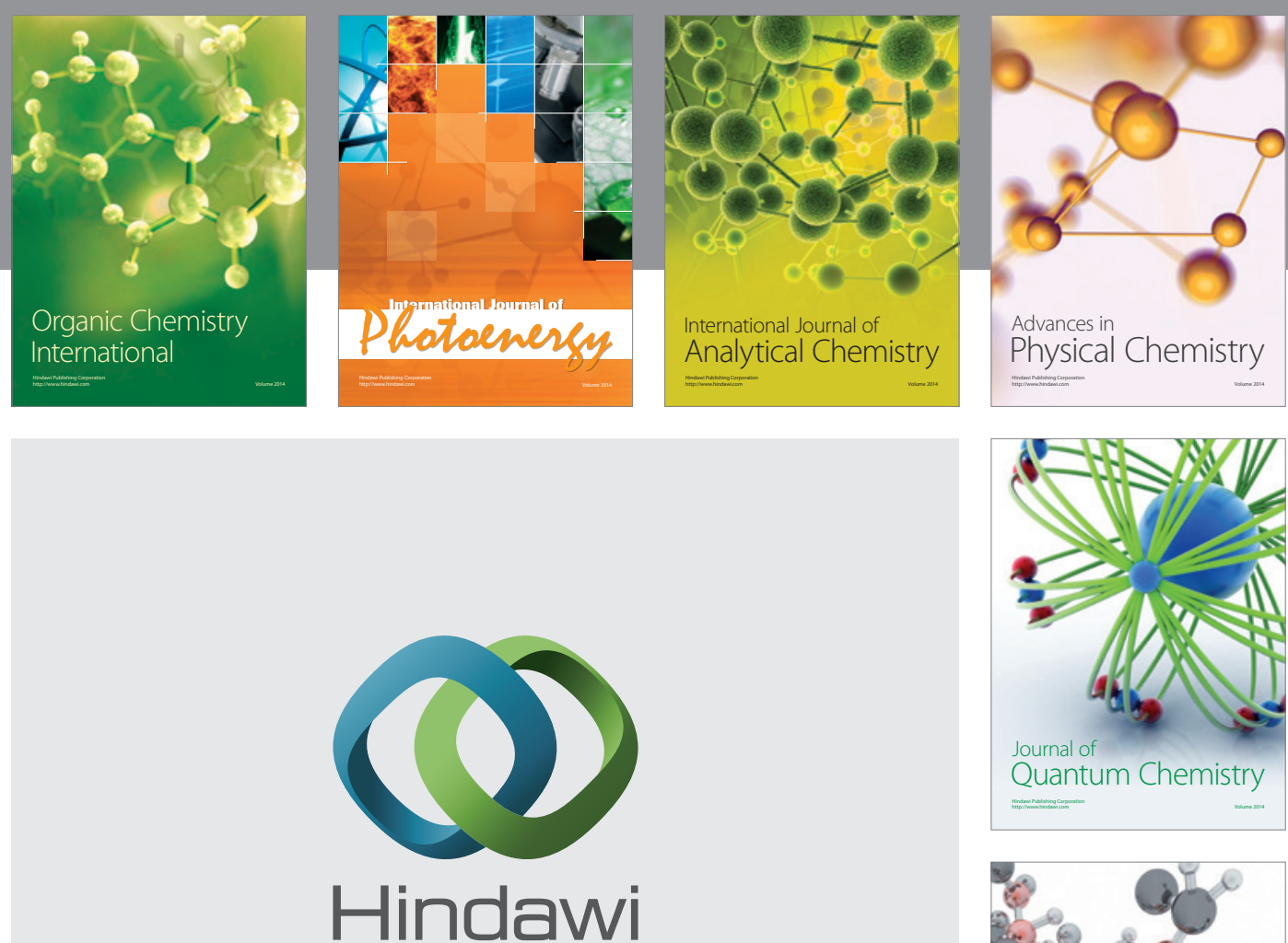

Submit your manuscripts at

http://www.hindawi.com

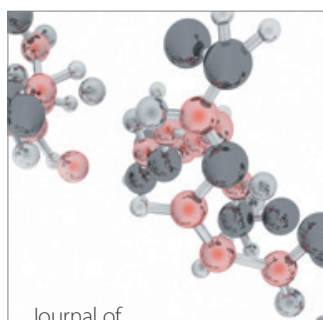

Analytical Methods

in Chemistry

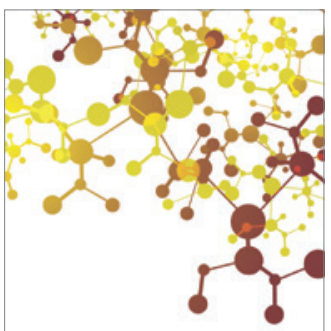

Journal of

Applied Chemistry

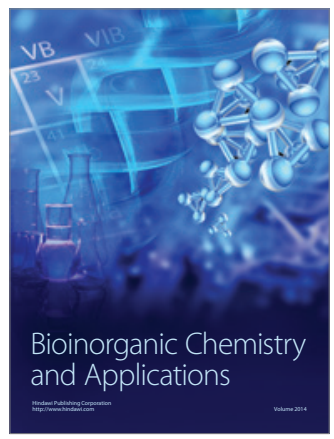

Inorganic Chemistry
